\title{
SOFT GAMMA REPEATERS REVISITED WITH BATSE
}

\author{
C. KOUVELIOTOU \\ USRA, NASA/MSFC, \\ ES-84, Huntsville, AL 35812, USA
}

\begin{abstract}
After the first 4 years of its operation, the Burst and Transient Source Experiment (BATSE) onboard the Compton Gamma Ray Observatory (CGRO), detected recurrent emission from two of the three known Soft Gamma Repeater (SGR) sources, SGR 1900+14 and SGR 1806-20. The reactivation of the latter prompted a quick international campaign resulting in the identification of the X-ray counterpart of the source, which also coincides with a compact radio source. The absence of detection of new sources in the 4 years of BATSE operation and the reactivation of two of the three already known SGRs, indicates that these objects are rare. We give here a short review of the previously detected SGR emissions and present the recent results obtained with BATSE.
\end{abstract}

\section{Introduction}

Of the over one thousand detected cosmic gamma-ray sources of transient emission, only three have clearly exhibited recurrent activity (Hurley 1986; Atteia et al. 1987; Laros et al. 1987; Kouveliotou et al. 1987). Apart from their recurrent nature, these events differ from the majority of gamma-ray bursts in the following characteristics:

- their temporal profiles often consist of single, simple pulses, usually with a triangular or trapezoidal shape;

- their durations are very short, typically of the order of $0.1 \mathrm{~s}$,

- their spectra are relatively soft, with characteristic temperatures in thermal-bremsstrahlung fits of the order of $20-30 \mathrm{keV}$.

The objects that emit these events can thus be considered as members of a distinct class - the Soft Gamma Repeaters (SGRs). 
The total number of events observed before BATSE from SGR 0526-66, SGR $1900+14$, and SGR $1806-20$, were 16,3 , and over 100 , respectively, with intervals between events that varied stochastically from hours to years (Norris et al. 1991). The embryonic SGR sky distribution suggested that they are galactic objects: the first source is located in the Large Magellanic Cloud and the remaining two are located in the Galactic plane (Norris et al. 1991). Two of the sources (SGR 1900+14 and SGR 1806-20) have been recently detected with BATSE (Kouveliotou et al. 1993, 1994); in the following sections we review these observations together with the previous activity of the sources.

\section{SGR $1900+14$}

\subsection{FIRST DETECTED ACTIVATION}

On 1979 March 24, 25 and 27, the KONUS experiments onboard the Venera 11-13 spacecraft recorded three soft transient events with very similar properties (Mazets et al. 1979); all three outbursts came from the same direction on the sky, centered at a rather large (almost 0.5 square degree) error box at R.A. $=286.47^{\circ}$ and $\operatorname{dec}=10.43^{\circ}$ (epoch 2000.0). In galactic coordinates the center of that region was located at $b^{\mathrm{II}}=1.6^{\circ}$ and $l^{\mathrm{II}}=$ $43.8^{\circ}$, very close to the Galactic plane.

TABLE 1. KONUS events from SGR $1900+14$

\begin{tabular}{|c|c|c|c|c|c|c|c|}
\hline $\begin{array}{l}\text { Date } \\
\text { Mar } 1979 \\
\text { day (UT) }\end{array}$ & $\begin{array}{l}\text { Dur. } \\
\text { ms }\end{array}$ & $\begin{array}{l}\text { Rise } \\
\text { time } \\
\text { ms }\end{array}$ & $\begin{array}{l}\text { Fluence } \\
10^{-6} \\
\text { erg cm }\end{array}$ & $\begin{array}{l}\text { Peak flux } \\
10^{-6} \\
\text { erg cm }{ }^{-2} \mathrm{~s}^{-1}\end{array}$ & $\begin{array}{l}\text { Peak } \\
\text { rate }^{\alpha} \\
\operatorname{cts}(15.6 \mathrm{~ms})^{-1}\end{array}$ & $\begin{array}{l}\Delta T^{\beta} \\
\text { hours }\end{array}$ & $\begin{array}{l}k T^{\gamma} \\
\mathrm{keV}\end{array}$ \\
\hline 24.6714 & 120 & $<8$ & 1.0 & 8.3 & 130 & - & $35 \pm 2$ \\
\hline 25.0822 & 190 & $<8$ & 1.5 & 7.9 & 80 & 9.9 & $36 \pm 2$ \\
\hline 27.4379 & 50 & $<8$ & 0.35 & 7.0 & 30 & 56.5 & $30 \pm 10$ \\
\hline
\end{tabular}

${ }^{\alpha}$ estimated from Fig. 1a

${ }^{\beta}$ time elapsed since previous event

${ }^{\gamma}$ from a thermal bremsstrahlung fit

The temporal profiles of the KONUS events are shown in Fig. 1a with a time resolution of $0.0156 \mathrm{~s}$. A feature that strikes the eye is the monotonic decrease of their peak count intensities: the first event is $\sim 1.5$ times more intense than the second, which in turn is $\sim 3.0$ times more intense than the third. The time separations of the events are $\sim 10$ and $\sim 56.5$ hours, respectively. Fig. 1b shows their spectra accumulated between $50-150 \mathrm{keV}$ and fitted with a thermal bremsstrahlung function; they are identical within 

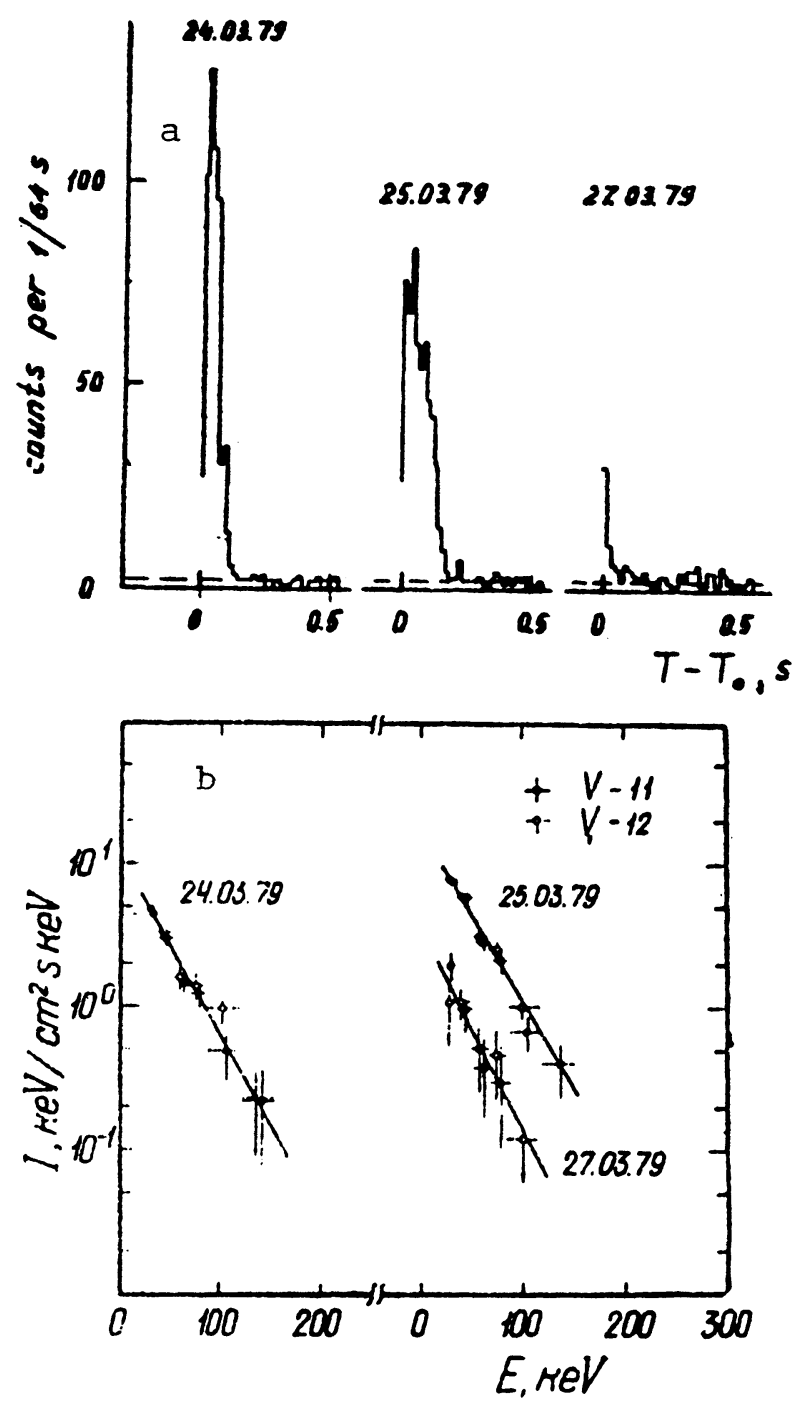

Figure 1. (a) Temporal profiles of the 3 outbursts of SGR $1900+14$ detected with the KONUS instrument in 1979, (b) Spectra from the same events (Mazets et al. 1979).

errors with an average $k T \sim 34 \mathrm{keV}$. Table 1 contains information of these early SGR $1900+14$ outbursts, such as durations, temperatures, fluxes, fluences, etc. From Table 1 we see that the average peak flux detected with KONUS was $7.710^{-6} \mathrm{erg} \mathrm{cm}^{-2} \mathrm{~s}^{-1}$. The rise times of $<8 \mathrm{~ms}$ are upper limits which reflect the instrument time resolution. 
According to Mazets et al. (1979), the KONUS instruments did not detect any other emission from this source; their post-factum search extended before the first trigger for a total period of 9 months (1978 September 1979 May). All in all the source burst for three times in March 1979.

\subsection{SECOND ACTIVATION}

On June 19, 1992 BATSE was triggered by a short, soft event clearly reminiscent of an SGR emission. The event was located near SGR $1900+14$, albeit with a large error box. The center of the error circle was at R.A. = $288^{\circ}$ and $\operatorname{dec}=11^{\circ}$ (epoch 2000.0), with a $\sim 5^{\circ} 1 \sigma$ radius. As repetition is a defining characteristic of an SGR, we decided to wait for the next outburst before we would alert the community. We had another reason to "wait it out": a new X-ray transient source, GRS $1915+105$, had just erupted in the vicinity (Castro-Tirado et al. 1992) and the BATSE error circle could not exclude it as the origin of the SGR event. In the next two months, BATSE recorded two more SGR-like outbursts coming from the part of the sky including the transient and SGR 1900+14. Fig. 2 shows the three location circles for these events, the BATSE contour map of the Aquila transient and the $2 \sigma$ "old" error box obtained from the KONUS events (epoch 2000.0). Although the BATSE data do not exclude GRS 1915+105 as the source of the triggered emission, the KONUS error box (which is known with arcmin accuracy) is $1^{\circ} .5$ away from the transient and cannot be related to it.

TABLE 2. BATSE events from SGR $1900+14$

\begin{tabular}{|c|c|c|c|c|c|c|c|}
\hline $\begin{array}{l}\text { Date } \\
1992 \\
\text { DOY (UT) }\end{array}$ & ms & $\begin{array}{l}\text { Rise } \\
\text { time } \\
\text { ms }\end{array}$ & $\begin{array}{l}\text { Fluence } \\
10^{-8} \\
\text { erg cm } \mathrm{cm}^{-2}\end{array}$ & $\begin{array}{l}\text { Peak flux } \\
10^{-6} \\
\text { erg cm } \text { cm }^{-2} \mathbf{s}^{-1}\end{array}$ & $\begin{array}{l}\text { Peak } \\
\text { rate }^{\alpha} \\
\operatorname{kcts}^{-1}\end{array}$ & $\begin{array}{l}\Delta T^{\beta} \\
\text { days }\end{array}$ & $\begin{array}{l}k T^{\gamma} \\
\mathrm{keV}\end{array}$ \\
\hline $171.7484 \mathrm{~A}$ & 40 & $<0.5$ & 4.5 & 1.1 & 40 & - & - \\
\hline 171.7484B & 40 & 15 & 4.3 & 1.0 & 100 & - & $39 \pm 3$ \\
\hline 190.2194 & $<64$ & $<64$ & 3.0 & 0.5 & 16 & 18.5 & - \\
\hline 232.2067 & 80 & $<5$ & 6.6 & 0.8 & 60 & 42.0 & - \\
\hline
\end{tabular}

${ }^{\alpha}$ from Kouveliotou et al. 1993

${ }^{\beta}$ time elapsed since previous event

$\gamma$ from a thermal bremsstrahlung fit

The overall properties of the bursts were very similar to the previous emissions from SGR $1900+14$ (see Table 2). Figs. 3a and 3b show the temporal structure of the first event recorded with $0.512 \mathrm{~ms}$ time resolution. This event was the longest of the three: it comprised two pulses separated by $\sim 0.5$ seconds with a total duration of $\sim 0.545 \mathrm{~s}$. The profiles of the pulses 


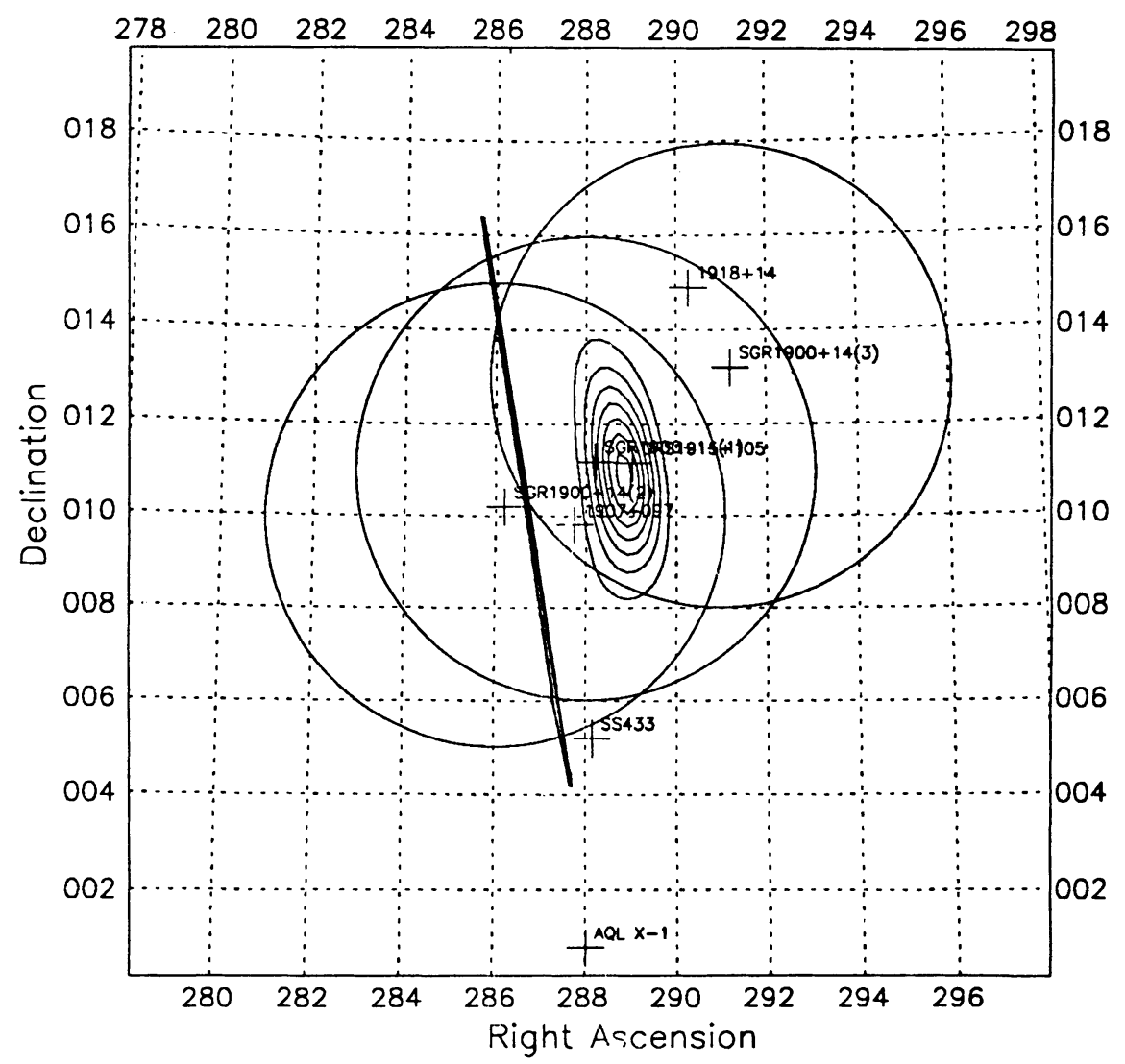

Figure 2. Sky-map of the area around SGR $1900+14$. The large circles are the three BATSE detections. The thin vertical box is the KONUS error box of 1979. The intensity contours are centered on the Aquila transient as detected with BATSE.

are trapezoidal and triangular, respectively. Each pulse is described with separate entries in Table 2, indicated with $\mathrm{A}$ and $\mathrm{B}$. We notice here that the rise time of the first pulse is well resolved and extremely small: $0.5 \mathrm{~ms}$; it is the shortest resolved rise time of an SGR event so far.

Fig. 3c shows the spectrum of the second pulse (the only one for which we had spectral data). The two lines designated by (1) and (2) in Fig. 3c correspond to best fits of an OTTB function to consequtive 16-ms time bins, with $k T=41 \pm 5$ and $36 \pm 3 \mathrm{keV}$, respectively; the spectra are identical within errors and thus show no spectral evolution during the pulse.

Conclusions: The combined KONUS-BATSE results indicate that activity from SGR $1900+14$ was detected again $\sim 13$ years after its discovery. 

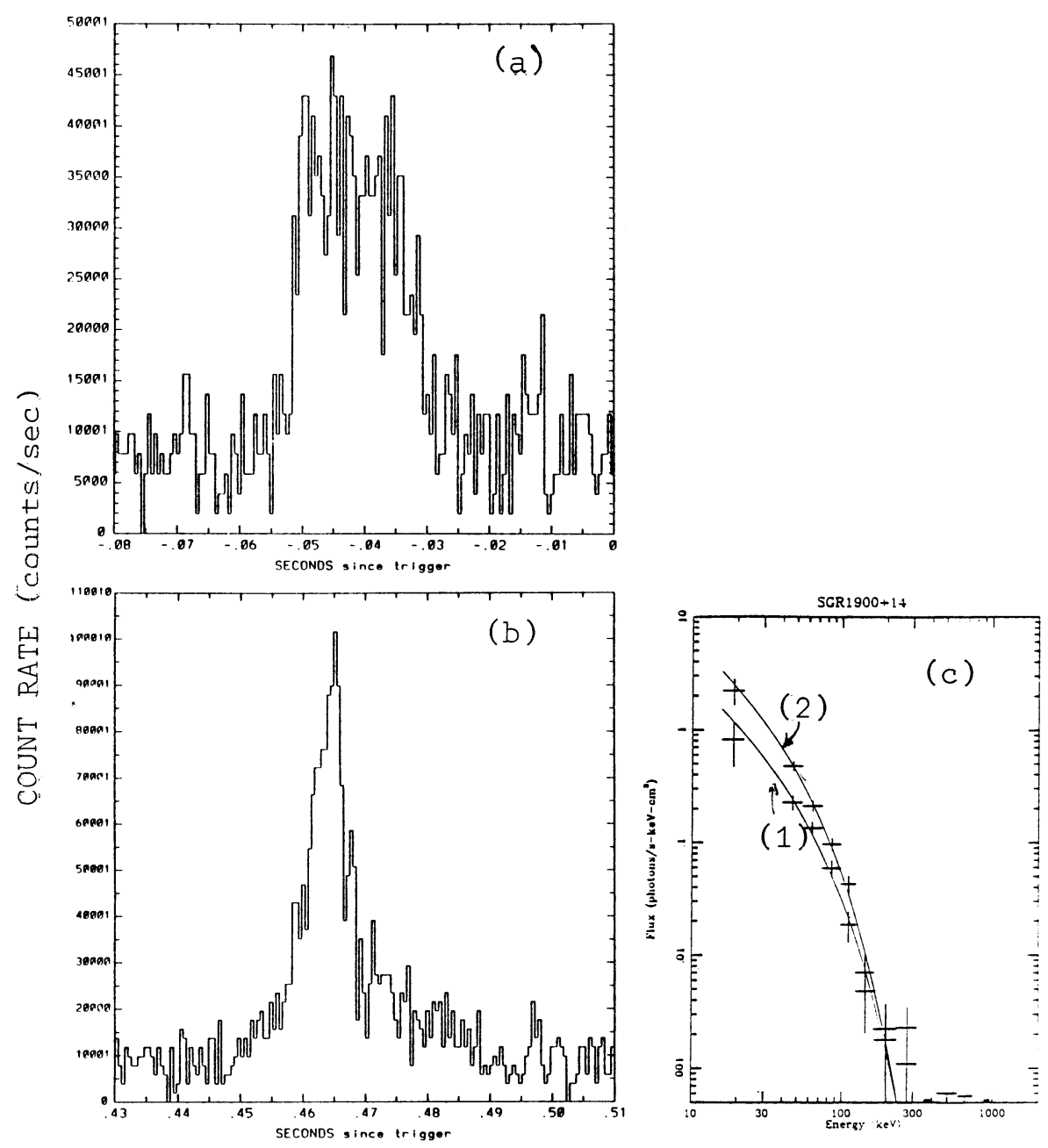

Figure 3. (a) Light curve of the first pulse of the 19 June 1992 trigger, (b) Second pulse of the same trigger. The counts are integrated between 20 and $100 \mathrm{keV}$. Time resolution is $0.512 \mathrm{~ms}$. (c) Photon spectrum for two consequtive 16-ms bins of the pulse in Fig. 3b. (1) the first 16-ms (2) the second 16-ms. The curves indicate the best-fit OTTB function, of the form $A \exp (-E / k T) / E$, with $k T=41 \pm 5$ and $36 \pm 3 \mathrm{keV}$, respectively.

The rise times were extremely short $(<0.5 \mathrm{~ms})$ and there was no spectral evolution within an event (at the 16-ms resolution). BATSE detected events with peak fluxes $\sim 10$ weaker than the previous detections. If we assume a galactocentric distance for the source, these fluxes would correspond to super-Eddington luminosities of $\sim 10^{41} \mathrm{ergs} \mathrm{s}^{-1}$. 

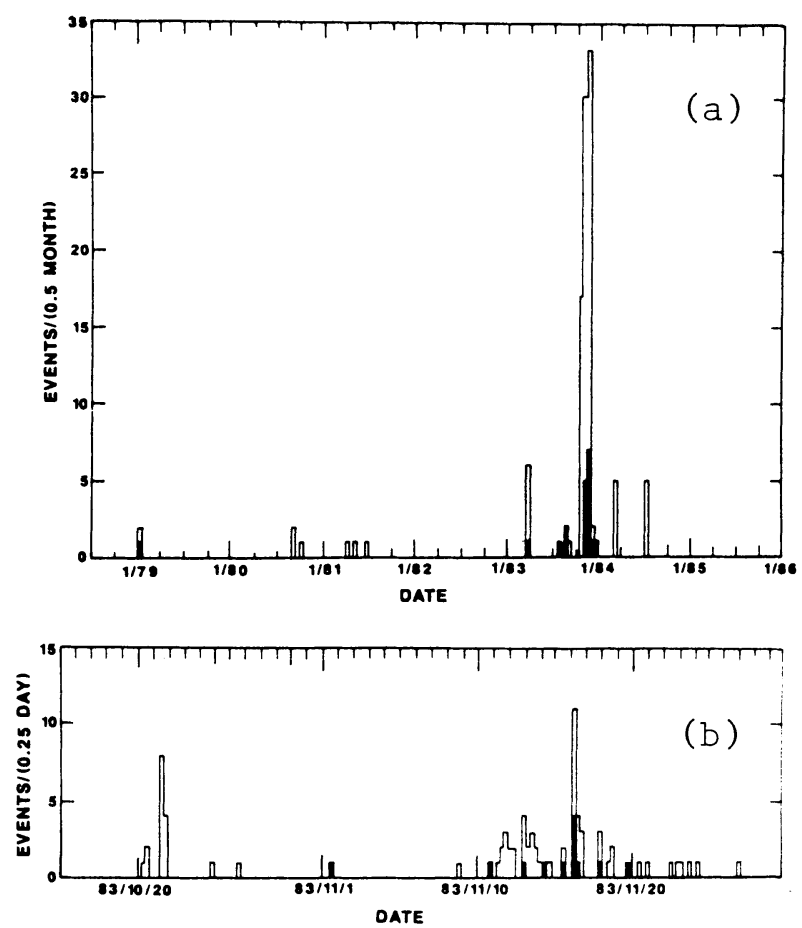

Figure 4. ICE events vs time. The filled-in part of each histogram is the subset of events also observed with other spacecraft. In (a) 8 years of data are shown at 0.5 month time resolution; (b) presents 1.5 months of data at 0.25 day resolution. The temporal coverage is $\sim 75 \%$ in $1978-1983$, and $\sim 20 \%$ thereafter (from Laros et al. 1987).

\section{SGR 1806-20}

\subsection{EARLIER DETECTIONS}

SGR 1806-20 was the last discovered repeater. Although the first emission of this source was immediately recognized as unusual due to the softness of its spectrum (Laros et al. 1986), it was later through the efforts of K. Hurley that subsequent emissions were associated with this source. In 1986, he discovered, while investigating the Prognoz satellite data, that several short, soft transient events appeared to come from the same general direction of the sky. A wide search effort (Atteia et al. 1987; Laros et al. 1987; Kouveliotou et al. 1987) to confirm this result, established 18, well-localized emissions with a common intersection at $(\alpha, \delta)_{1950.0}=\left(18^{\mathrm{h}} 06^{\mathrm{m}},-20^{\circ}\right)$. In addition, between 1978 August 13 and 1986 June 27, 111 events were assigned with high probability to the repeater (see Fig. 4) based on their SGR-like properties, although most were observed only with one instrument, the International Cometary Explorer (ICE) (Laros et al. 1987). 
The statistical properties of these 111 emissions have been studied extensively by Laros et al. (1987). They find little or no correlation between event arrival times and intensities and that clustering persists on shorter time scales (intervals range from $1 \mathrm{~s}$ to $>1$ year). In fact, for the very weak bursts $(\log I<1.4), 12$ of 15 events are associated with intervals shorter than the median interval of $1400 \mathrm{~s}$, while the most intense bursts apparently have no preferred separations (Laros et al. 1987). The event spectra are best fit with an OTTB function with temperatures between 24 and $40 \mathrm{keV}$; there are strong indications for a spectral turnover below $10 \mathrm{keV}$. The range in the burst intensities spans a factor of 30; the $\log N-\log P$ function can be approximated by a power law that flattens at low luminosities. The average peak flux of these bursts was $\sim 3.210^{-6} \mathrm{ergs} \mathrm{cm}^{-2} \mathrm{~s}^{-1}$ (Norris et al. 1991).

\subsection{BATSE DETECTIONS OF SGR $1806-20$}

On 29 September 1993, BATSE detected three bursts from SGR 1806-20 within 14 hours; the source appeared again on 5 Oct, on 9 Oct, and on 10 Nov 1993, respectively (Kouveliotou et al. 1994). Four of the six bursts have simple, triangular profiles. The third event on 29 September consisted of two brief pulses separated by one second, closely resembling a previous emission from the source in 1983 (Kouveliotou et al. 1987). Table 1 from Kouveliotou et al. (1994) has all the pertinent information on the properties of these outbursts. We note here that their peak fluxes range between 2 and $910^{-6}$ ergs $\mathrm{cm}^{-2} \mathrm{~s}$, values which are 4 to 15 times smaller than those of the brightest events detected from this source (Norris et al. 1991) and comparable to the ones detected with the Solar Maximum Mission (SMM) (Kouveliotou et al. 1987). The intervals between these 6 bursts range from 0.1 to 31.5 days.

We have derived a spectrum for the third burst of 29 September 1993, over $4.096 \mathrm{~s}$ from background-subtracted 16 energy channel data. A fit with an optically-thin thermal bremsstrahlung (OTTB) function yields a value of $k T=28 \pm 5 \mathrm{keV}$, and a normalization of $1.8 \pm 0.6$ photons $\left(\mathrm{s}^{-1} \mathrm{keV}^{-1} \mathrm{~cm}^{-2}\right)$ at $1 \mathrm{keV}$. The $k T$ value is similar to the ones reported before (Atteia et al. 1987; Kouveliotou et al. 1987; Laros et al. 1986; Fenimore et al. 1993), indicating very little change in the emission mechanism over 10 years. The fluences cluster around an average value of $\sim 10^{-7} \mathrm{erg} \mathrm{cm}^{-2}$.

Fig. 5 shows the overlapping error circles of the 6 bursts, drawn with the $1 \sigma$ radius of $8^{\circ}$. The very narrow diamond indicated by $I$ is the updated (K. Hurley, personal communication) SGR 1806-20 location (epoch 2000.0) computed with data from the 2nd Inter-Planetary Network (IPN). Clearly, the BATSE circles overlap the IPN location.

Conclusions: SGR 1806-20 has been found active again $\sim 14$ years after 


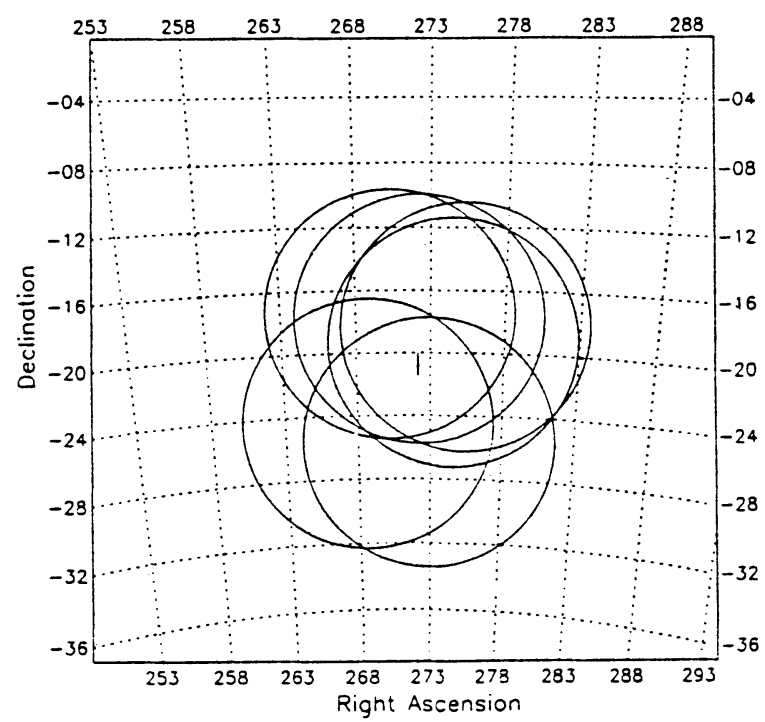

Figure 5. Sky map of the region near $\alpha=271^{\circ}, \delta=-20^{\circ}$. The six large circles represent the locations of the BATSE events. The size of the circles $\left(8^{\circ}\right)$ reflects mainly the statistical errors of the measured positions. Burst locations are obtained from the relative strength of their signals in the relevant subset of the 8 identical BATSE Large Area Detectors (LADs). The position determination includes the detailed spectral dependence of the angular sensitivity of the detectors, and corrections for photon back-scattering by the Earth's atmosphere. The narrow diamond (I) represents the $2 \sigma$ error box of SGR 1806-20 (K. Hurley, private communication).

its discovery. BATSE has detected outbursts 4 to 15 times weaker than the brightest KONUS events. For distances in the range 8.5 to $17 \mathrm{kpc}$ (Kulkarni \& Frail 1993) the peak luminosity for the brightest BATSE events is between $710^{40}$ and $310^{41} \mathrm{ergs} \mathrm{s}^{-1}$, i.e., the events are highly super-Eddington if they are unbeamed and originate from neutron stars.

\section{Counterpart searches}

Based on the identification of all three emissions on 29 September with SGR 1806-20, the BATSE team initiated an international multi-wavelength campaign to search for counterparts in the IPN SGR error box. As a result, on 9 October 1993 the Japanese X-ray satellite ASCA detected (Murakami et al. 1994) a burst from the area of the source simultaneous with BATSE. ASCA also discovered (Murakami et al. 1994) the steady X-ray counterpart of SGR 1806-20.

Earlier searches (Kulkarni \& Frail 1993) had suggested the identification of SGR 1806-20 with G 10.0-0.3, a SNR which was later identified to be of a rare type, known as a plerion (also known as "filled-center" or "Crab-like" 


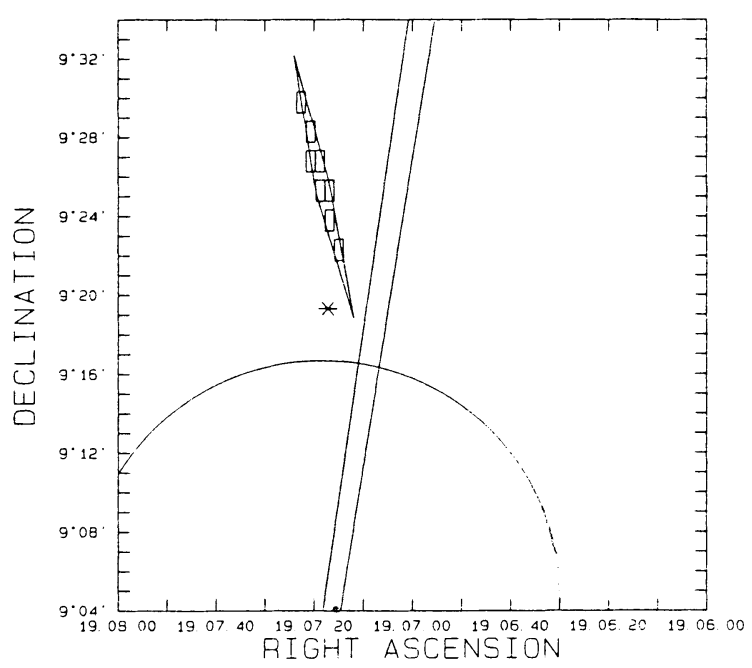

Figure 6. Map of the region around SGR $1900+14$. The two vertical lines are the boundaries of the KONUS error box (see also Fig. 2), the diamond with the rectangles is the virtual network synthesis error box, the circle encompasses the radio contours of G 42.8+0.6, and the asterisk is at the position of the ROSAT source mentioned in the text (from Hurley et al. 1994).

SNRs). Plerions are young SNRs which have centrally brightened morphologies, and are powered by a central object (known to be a pulsar in the case of the Crab nebula). SGR 0526-66 has been (controversially) associated with a SNR (N49) in the Large Magellanic Cloud (Cline 1981). Although this remnant is not a plerion, it is relatively young (Vancura et al. 1992) and could contain a pulsar.

Given the apparent radio and optical associations found for the other two SGRs, we searched for currently catalogued SNRs (Green 1991; Reich et al. 1990) in the error box of SGR $1900+14$, and found three that are overlapped by this error box: G 43.9+1.6, G 42.8+0.6 and G 39.7-2.0 (= W50). Recently, Hurley et al. (1994) have developed a virtual network synthesis method which enabled them to reduce the $430 \mathrm{arcmin}^{2}$ error box of SGR $1900+14$ by a factor of 70 . Fig. 6 shows this smaller error box for the SGR together with the "old" KONUS error box and the outer contours of G 42.8+0.6. Searching in that region Vasisht et al. (1994) have identified a ROSAT source which is very close to both objects (SGR and SNR); it is thus a prime candidate for the counterpart and several planned future observations will shed light on the nature of this object. 


\section{Discussion}

From the fact that reactivation of two of the three known SGRs has been observed with BATSE, but that no new SGRs have been detected, it follows that the total number, $N_{\mathrm{SGR}}$, of SGR sources in the Galaxy with properties similar to those already known, (including repetition time scales), is probably very limited. A precise limit is difficult to derive in view of gaps in monitoring as well as the differences in sensitivity, sky exposure and trigger criteria of the various experiments capable of observing SGRs. We have estimated a rough upper limit on $N_{\mathrm{SGR}}$ by assuming approximately equal sky exposures of BATSE and the experiments with which the SGR sources were detected ( $\sim 12$ and $\sim 13$ ster yrs, respectively). For a series of assumed values of $N_{\mathrm{SGR}}$ we calculated the probability that at least two of the known sources are detected again with BATSE, but no new SGR sources are detected, and from this we infer that $N_{\mathrm{SGR}}<7$ (95\% level). In view of the above mentioned uncertainties we suggest that it is unlikely that our Galaxy contains at any given time more than a few active SGR sources.

Our detections show that SGRs keep their ability to be active for many years. The extended duration of SGR activity strengthens the argument that these sources are related to galactic (likely Population I) objects, quite plausibly neutron stars (Kouveliotou et al. 1987; Norris et al. 1991), as recurrent SGR emissions do not signify a unique (catastrophic) event in the life cycle of the source. The association of SGR 1806-20 with a plerion suggests that the events originate from young neutron stars, with ages less than $\sim 10^{4}$ years. With neutron star birth rates of order 1 per 100 years (Strom 1994; Lorimer et al. 1993) this would imply that either the active SGR phase lasts less than $\sim 10^{3}$ years, or that only a small fraction $(\sim 10$ percent) of these young neutron stars become SGR sources. It is currently unknown what special property would turn a young neutron star into an SGR source.

\section{References}

Atteia, J.-L. et al. 1987, ApJ 320, L105

Castro-Tirado, A.J. et al. 1992, IAU Circular 5590

Cline, T.L. 1981, Ann. NY Acad. Sci. 375, 314

Green, D.A. 1991, PASP 103, 209

Fenimore, E.E., Laros, J.G. \& Ulmer, A. 1993, ApJ 418, 395

Hurley, K. 1986, talk presented at Taos Gamma Ray Stars Conference

Hurley, K. et al. 1994, ApJ 431, L31

Kouveliotou, C. et al. 1987, ApJ 322, L21

Kouveliotou, C. et al. 1993, Nat 362, 728

Kouveliotou, C. et al. 1994, Nat 368, 125

Kulkarni, S. \& Frail D.A. 1993, Nat 365, 33 
Laros, J.G. et al. 1986, Nat 322, 152

Laros, J. et al. 1987, ApJ 320, L111

Lorimer, D.R. et al. 1993, MNRAS 263, 403

Mazets, E.P., Golenetskii, S.V., Guryan, Yu.A. 1979, SvA Lett. 5 (6), 343

Murakami, T. et al. 1994, Nat 368, 127

Norris, J.P. et al. 1991, ApJ 366, 240

Reich, W. et al. 1990, A\&AS 85, 633

Strom, R. 1994, in The Lives of the Neutron Stars, M.A. Alpar, Ü. Kızloğlu \& J. van Paradijs (Eds.), NATO ASI Vol. C450, p. 23

Vancura, O. et al. 1992, ApJ, 394, 158

Vasisht, G. et al. 1994, ApJ 431, L35 